\title{
Numerical Analysis for Water-Exit Trajectory of Submarine-launched UUV
}

\author{
Yonghu Wang ${ }^{1,2}$, Tianlong Lin ${ }^{1, *}$ and Hao Jiang ${ }^{1}$ \\ ${ }^{1}$ Flight Technology College, Civil Aviation Flight University of China, Guanghan 618307, Sichuan, China \\ ${ }^{2}$ Wenzhou University, Wenzhou 325035, Zhejiang, China \\ ${ }^{*}$ Corresponding author
}

\begin{abstract}
In order to completely perform the mission, It is significant to study the trajectory of projectiles, e.g. Unmanned Underwater Vehicle (UUV) in the process of submarine launch. Three dimensional numerical model is established based on the volume of fluid (VOF) method in combination with the realizable $k-\varepsilon$ viscous model, open channel model, and dynamic grid technology. Six degrees of freedom (6DOF) trajectory were realized quantitatively in the simulation process under three cases, including that static flow and water flow with constant velocity and various velocity. At the same time, the pressure field is analyzed in the whole launch process. It concludes that the pressure field only changes around the UUV's head cap and tail part. The influences of constant velocity on dynamic characteristics of UUV are different from various velocity. Water velocity is no negligible impact factor which decide whether the UUV launch successfully.
\end{abstract}

Keywords-UUV; water exit; the VOF method; trajectory analysis; dynamic grid; numerical simulation

\section{INTRODUCTION}

It is a complex process of the submarine-launched projectile when it across from the different media, that is water and air. And it is a structure, gas and water multiphase coupling problem. The underwater trajectory of UUV includes tube-exit stage, free moving stage in water and water-exit stage. During the moving process, the position and trajectory of UUV are affected by the water flow conditions, causing the projectile to deviate from the ideal trajectory [1]. Numerical simulation of submarine-launched projectile's launching and flow field was carried out [2]. The computational fluid dynamics (CFD) method is used to simulate the process of missile exiting from water by solving the Navier-Stokes equations. The free-surface problem is an important issue in fluid mechanics research. The multiphase flow model uses the free-surface tracking method such as MAC, VOF and Level-set methods [3]. The interface can deal with the flow problem where the free interface changes drastically but the interface is basically clear.

In this paper, three-dimensional moving process of submarine-launched UUV is simulated in the framework of ANSYS FLUENT codes which adopt the realizable viscous model, utilize dynamic grid and six degrees of freedom model. In order to capture the free water surface, the VOF method is used to simulate well. The whole moving process of three cases is numerically simulated in order to study the influence of various water velocities on the launched trajectory on details.

\section{THEORETICAL METHOD}

\section{A. VOF Method}

The VOF model can calculate a multiphase flow by solving a set of momentum equations and tracking the volume fraction of each fluid in the region [4]. The VOF equation is mainly applicable to two or more incompatible fluids.

The volume fraction of qth fluid in the grid is expressed as $\alpha_{q}$, then $\alpha_{q}=0$ means that the $q$ th fluid of grid is empty, $\alpha_{q}=1$ represents the grid filled with qth fluid, $0<\alpha_{q}<1$ represents grid $q$ in the fluid and other fluids. Discrete formula of volume fraction is

$$
\frac{\alpha_{q}^{n+1} \rho_{q}^{n+1}-\alpha_{q}^{n} \rho_{q}^{n}}{\Delta t} V+\sum_{f} \rho_{q} U_{f}^{n} \alpha_{q, f}^{n}=\left[\sum_{p=1}^{n}\left(\dot{m}_{p q}-\dot{m}_{q p}\right)+S_{\alpha_{q}}\right] V
$$

\section{B. Open Channel Model}

The open channel model [5] is generally used to set the flow rate of water. It may prevent the free surface from collapsing. The free water surface and the bottom surface are set the pressure outlet. The pressure regulation used is free flow to process density interpolation method.

Free surface $y_{\text {local }}$ in the control equation is

$$
\mathrm{y}_{\text {local }}=-(\vec{a} \cdot \hat{g})
$$

Here, $\vec{a}$ is the position vector of any point on the free surface, and $\hat{g}$ is the unit vector in the direction of gravity.

\section{Dynamic Grid Method}

Dynamic grids are generally used to grasp the dynamics of the flow field and track the dynamics of the boundary over time [6].In this paper, the approximate spring smoothing model is selected and the local reconstruction model of the grid is performed, in which the elastic constant is set to 0.5 , the grid redraw method is applied to the local grids and local planes, and the size function is given. The other coefficients are always 
the default values. UDFs (user defined functions) are used to define the mass and moment of inertia of UUV.

In the inertial coordinate system, the center of gravity translation motion control equation is:

$$
\dot{\vec{v}}_{G}=\frac{1}{m} \sum \vec{f}_{G}
$$

Here, $\vec{v}_{G}$ is the translational movement of the center of gravity, $m$ is the mass of the cylinder, and $\vec{f}_{G}$ is the vector of gravity.

\section{Maintaining the Integrity of the Specifications}

The realizable $k-\varepsilon$ viscous model contains an alternative formula for the turbulent viscosity. In the modified transport equation, the turbulence dissipation rate $\varepsilon$ comes from the exact equation for the fluctuation of squared vorticity.

The transport equation is:

$$
\begin{aligned}
& \frac{\partial}{\partial t}(\rho k)+\frac{\partial}{\partial x_{j}}\left(\rho k u_{j}\right)=\frac{\partial}{\partial x_{j}}\left[\left(\mu+\frac{\mu_{t}}{\sigma_{k}}\right) \frac{\partial k}{\partial x_{j}}\right]+ \\
& G_{k}+G_{b}-\rho \varepsilon-Y_{M}+S_{k}
\end{aligned}
$$

and

$$
\begin{aligned}
& \frac{\partial}{\partial t}(\rho \varepsilon)+\frac{\partial}{\partial x_{j}}\left(\rho \varepsilon u_{j}\right)=\frac{\partial}{\partial x_{j}}\left[\left(\mu+\frac{\mu_{t}}{\sigma_{\varepsilon}}\right) \frac{\partial \varepsilon}{\partial x_{j}}\right]+\rho C_{1} S \varepsilon- \\
& \rho C_{2} \frac{\varepsilon^{2}}{k+\sqrt{v \varepsilon}}+C_{1 \varepsilon} \frac{\varepsilon}{k} C_{3 \varepsilon} G_{b}+S_{\varepsilon}
\end{aligned}
$$

Here,

$$
\begin{gathered}
C_{1}=\max \left[0.43, \frac{\eta}{\eta+5}\right] \\
\eta=S \frac{k}{\varepsilon} \\
S=\sqrt{2 S_{i j} S_{i j}}
\end{gathered}
$$

Where, $G_{k}$ denotes the gradient turbulent kinetic energy from the average velocity, $G_{b}$ represents the turbulent kinetic energy produced by buoyancy. $Y_{M}$ is the swelled value of the compressible flow fluctuations, $C_{2}$ and $C_{1 \varepsilon}$ are constants, $\sigma_{k}$ and $\sigma_{\varepsilon}$ are the turbulence Prandtl numbers for $k$ and $\varepsilon$.

\section{E. Other Methods}

SIMPLC algorithm is applied to solve the pressure and velocity coupling, the least squares method to solve the gradient equation, PRESTO! to solve the pressure difference, and volume reconstruction to solve the volume fraction, the other are second-order upwind solution.

\section{NUMERICAL SimUlATION METHOD}

According to the above theoretical model, the numerical simulation of submarine-launched UUV from the outlet tube is carried out. The UUV is 9 meters long and weighs 20 tons. The rotational inertia and parameters of the missile are shown in Table1.

TABLE I. THE PARAMETERS OF UUV

\begin{tabular}{|c|c|}
\hline Physical parameters & - \\
\hline$I_{x x}$ & $6.48009 \times 10^{5} \mathrm{~kg} \cdot \mathrm{m}^{2}$ \\
\hline$I_{y y}$ & $6.48105 \times 10^{5} \mathrm{~kg} \cdot \mathrm{m}^{2}$ \\
\hline$I_{z z}$ & $2.0296 \times 10^{3} \mathrm{~kg} \cdot \mathrm{m}^{2}$ \\
\hline Center of gravity & $(0.0696,0,-5.263)$ \\
\hline
\end{tabular}

The UUV is launched from 30 meters underwater. The launching speed of the projectile is $30 \mathrm{~m} / \mathrm{s}$. The water flow is shown in Table 2

TABLE II. THE SPEED OF WATER FLOW

\begin{tabular}{|c|c|}
\hline & velocity $(\boldsymbol{m} / \mathbf{s})$ \\
\hline CASE 1 & $\mathrm{~V} 1$ \\
\hline CASE 2 & $\mathrm{~V} 2$ \\
\hline CASE 3 & $\mathrm{~V} 3$ \\
\hline
\end{tabular}

$$
V_{1}=0 m / s, V_{3}=2 m / s, V_{2}=\left\{\begin{array}{cc}
0 & z \leq 0 \\
0.04 \times z+1 & 0<z \leq 15 \\
0.04 \times z+1.3 & 15<z \leq 25 \\
0.04 \times z+0.8 & z>25
\end{array}\right.
$$

In the paper, three-dimensional numerical water pool is established in ICEM CFD 16.0 program. The pool height, length and width are $60 \mathrm{~m} \times 120 \mathrm{~m} \times 60 \mathrm{~m}$. The global mesh of the numerical model is an unstructured tetrahedral mesh, and density box is used to mesh the structure. In order to save the computation time, coarse grids are used in the fluid-solid coupling area. The boundary condition of the flow inlet is set as a velocity inlet, water outlet is the pressure outlet, and the top of numerical pool is a pressure outlet and its bottom is wall. 


\section{ANALYSIS AND DISCUSSION}

When the submarine-launched UUV moves away form the deck of the launch tube, the entire flow field will change from time to time, especially for the water pressure. From the Figure 1 to Figure 3, it can be seen that the UUV's movement has little influence on the pressure distribution of the whole flow field. The influence domain is only limited to the water field around the UUV. A larger pressure value is in the water domain around the head of the UUV. With the increasing of the moving displacement, the velocity of UUV continues to decrease, and the pressure value of UUV's head cap go down continually.

The trajectory of UUV is shown in Figure 4, and the purpose is to analyze the water flow impact on the trajectory. Comparing the trajectories of CASE2 to CASE3, the former deviates slightly, and the deviation of CASE3 is slightly the largest.
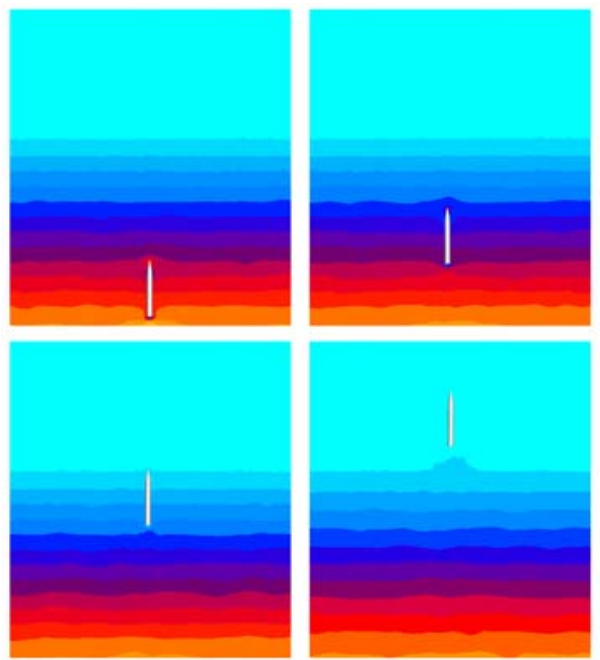

FIGURE I. THE PRESSURE FIELD OF CASE 1
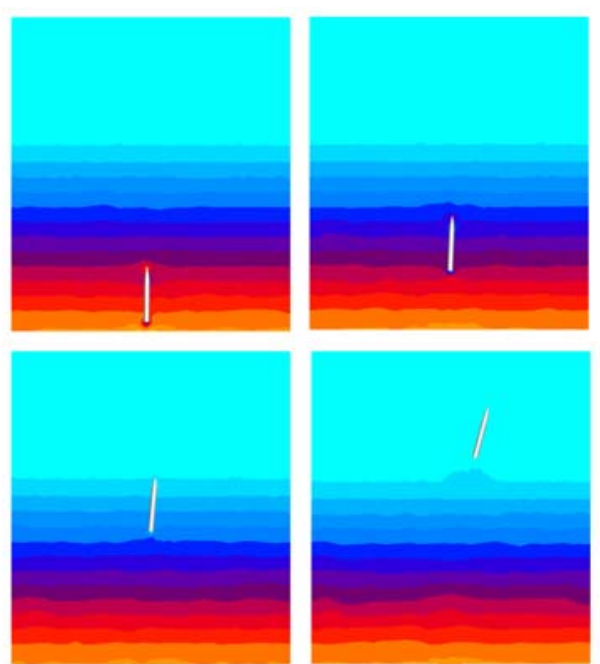

FIGURE II. THE PRESSURE FIELD OF CASE 2
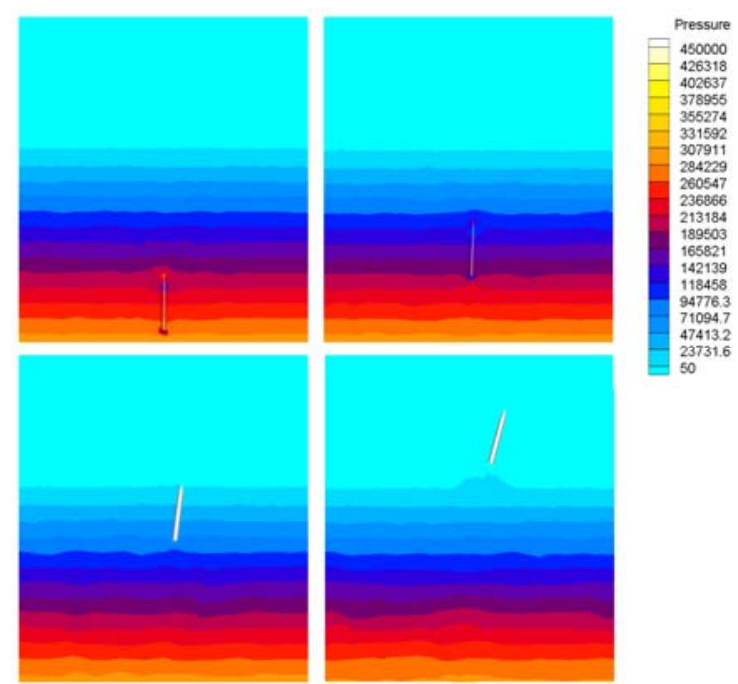

FIGURE III. THE PRESSURE FIELD OF CASE 3

When $z=25 m$, the UUV just touches the water surface. After the UUV move out of water level, the trajectory of the UUV gradually tends to be gentle. And the same time, the velocity decreases gradually. When $\mathrm{z}=30 \mathrm{~m}$, the UUV completely exits water level, the acceleration value gradually becomes $9.81 \mathrm{~m} / \mathrm{s}^{2}$.

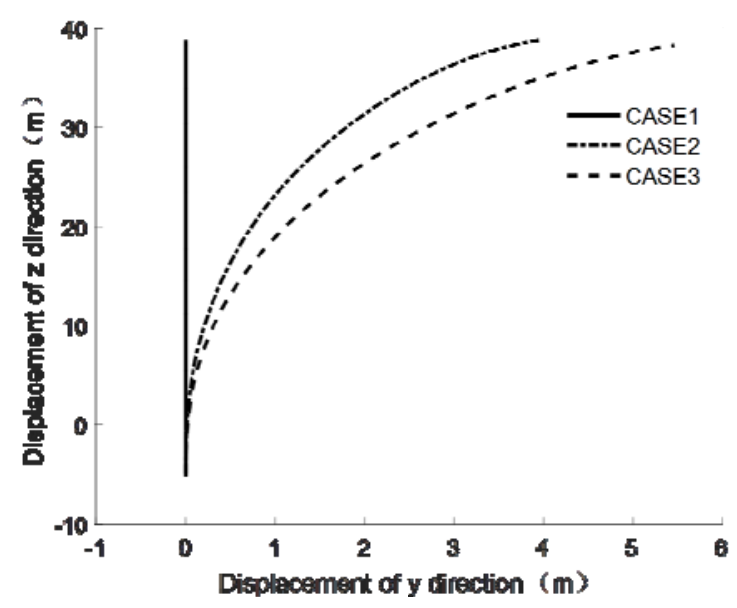

FIGURE IV. THE TRAJECTORY OF UUV

Figure 5 shows the change of velocity in y directions in the whole process. It can be seen that the velocity of UUV in CASE3 reaches $2 \mathrm{~m} / \mathrm{s}$ which is bigger than the one in CASE2, and the acceleration of UUV in CASE3 is bigger than in CASE2 when the time is less than 1 second. The effect of water flow on UUV in CASE3 is greater than in CASE2. The main reason is that the flow of water in the third condition is greater than the second one. The displacement of CASE3 is about 2 meters longer than CASE2's in the y direction. If the max flow velocity in CASE3 is equal to CASE2 and their flow direction is the same, the former will make UUV's horizontal velocity to reach $2 \mathrm{~m} / \mathrm{s}$ earlier than the latter. The constant water flow has influences of on dynamic characteristics of UUV more than other cases. 


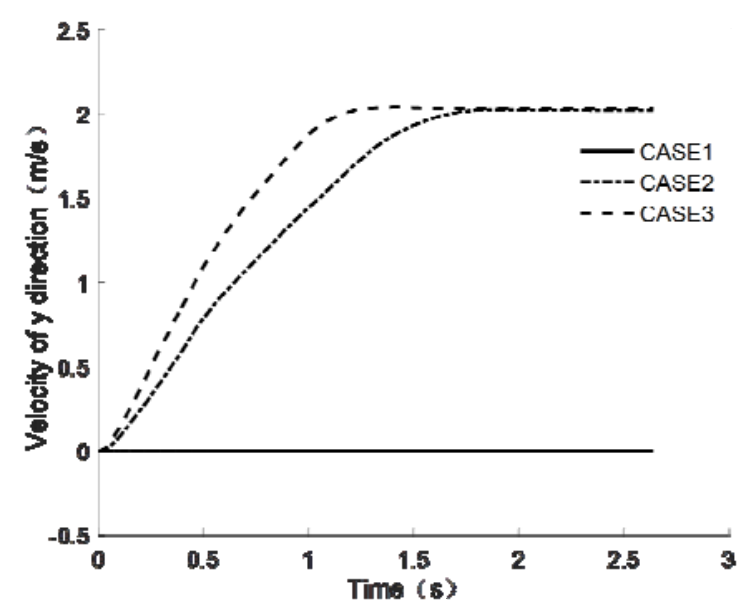

FIGURE V. THE VELOCITY - TIME HISTORY DIAGRAM

\section{CONCLUSION}

Computational Fluid Dynamics method is performed to simulate the submarine-launched process of the UUV based on VOF method and the introduction of the Open Channel Model. The main results of the research are emphasized as follows.

(1) Open Channel Model is a very effective method to simulate water flow in the application of water exit problem.

(2)The moving process of the UUV has little effect on the pressure distribution of the entire flow field. The pressure around the head and tail of the UUV are changed greatly.

(3) Comparing with the water-exit problem in clam water, constant flow velocity and various velocities will cause the projectile move in the horizontal displacement.

(4) With the consideration of the influence of different gradient flows, it is not impossible to make use of the flow direction and velocity and select the proper time and position to effectively launch the projectiles. It may improve the ability of the submarine-launched projectile to perform the mission.

\section{ACKNOWLEDGMENT}

This research was supported by the National Science Foundation (Grant No.U1733203) and Grant No.16ZB0030 of Sichuan Provincial Education Department and Grant No. J2016-65 of CAFUC.

\section{REFERENCES}

[1] Honjun ZHANG, Hongzhi LU, Yin PEI, Jian YE, Zhengping ZOU. Numerical Investigation to Vertical Launching missile. Chinese Journal of Hydrodynamics. 2010, 25(3):406-415.

[2] Weiguo ZHONG, Jiazhong ZHANG. Numerical simulation of underwater launched vehicle's submarine trajectory. Journal of ballistics, 2005, 17(1):8-12.

[3] HIRT C W. Volume of fluid (VOF) method for the dynamics of free boundaries. J Comput Phys, 1981, 39: 201-225.

[4] Yile LI, Yingzhong LIU, Guoping LIAO. Second Order Free Surface Tracking Algorithm in VOF Method and Its Application. Journal of Ship Mechanics, 1999, 3(01):44-52.

[5] Jing LU, Xiangdong WANG, Jianzhao GUAN, Zhaoyan WANG. Verification of 3-D Numerical Model for Open-channel Flows Based on Fluent. Science Technology and Engineering, 2012, 12(32):8579-8582.
[6] Zhongjun SHI, Min XU, Shilu CHEN. Analysis of Moving Mesh Generation Technology. Journal of Air Force Engineering University (Natural Science Edition), 2003, 4(01):61-64. 